\title{
Probing Competitive Interactions in Quaternary Formulations
}

Omar T. Mansour, ${ }^{\mathrm{a}}$ Beatrice Cattoz, ${ }^{\mathrm{a}}$ Richard K. Heenan, ${ }^{\mathrm{b}}$ Stephen M. King ${ }^{\mathrm{b}}$ and Peter C. Griffiths ${ }^{\mathrm{a}}{ }^{*}$

a Faculty of Science and Engineering, University of Greenwich, Medway Campus,

Central Avenue, Chatham Maritime, Kent ME4 4TB

b Science and Technology Facilities Council, ISIS Facility, Rutherford Appleton Laboratory, Didcot, Oxfordshire OX11 OQX, UK

*Corresponding author: Tel: +44 02083319927

Email address: p.griffiths@greenwich.ac.uk

\begin{abstract}
Hypothesis: The interaction of amphiphilic block copolymers of the poly(ethylene oxide)poly(propylene oxide)-poly(ethylene oxide) (PEO-PPO-PEO) group with small molecule surfactants may be "tuned" by the presence of selected alcohols, with strong interactions leading to substantial changes in (mixed) micelle morphology, whilst weaker interactions lead to coexisting micelle types.

Experiments: The nature and the strength of the interactions between Pluronic P123 $\left(\mathrm{EO}_{20} \mathrm{PO}_{70} \mathrm{EO}_{20}\right)$ and small molecule surfactants (anionic sodium dodecylsulfate, SDS, $\mathrm{C}_{12} \mathrm{SO}_{4} \mathrm{Na}$ ), (cationic dodecyltrimethylammonium bromide, $\mathrm{C}_{12} \mathrm{TAB}$ ) and (non-ionic polyoxyethylene(23)lauryl ether, Brij $35, \mathrm{C}_{12} \mathrm{EO}_{23} \mathrm{OH}$ ) is expected to depend on the partitioning of the short, medium and long chain alcohols (ethanol, hexanol and decanol respectively) and was probed using tensiometry, pulsed-gradient spin-echo nuclear magnetic resonance (PGSE-NMR) and small-angle neutron scattering (SANS). Findings: The SANS data for aqueous P123 solutions with added alcohols were well described by a charged spherical core/shell model for the micelle morphology. The addition of the surfactants led to significantly smaller, oblate elliptical mixed micelles in the absence of alcohols. Addition of ethanol to these systems led to a decrease in the
\end{abstract}


micelle size, whereas larger micelles were observed upon addition of the longer chain alcohols. NMR studies provided complementary estimates of the micelle composition, and the partitioning of the various components into the micelle.

Keywords: Pluronic micelle, small molecule surfactant, PGSE-NMR, SANS

\section{Introduction}

Amphiphilic molecules, commonly known as surfactants, form discrete aggregates, called micelles above the critical micelle concentration (CMC). A widely studied and practically relevant series of surfactants is the water soluble triblock copolymer group made up of poly(ethylene oxide) (PEO) and poly(propylene oxide) (PPO), denoted as (PEOn-PPOm-PEOn), commercially known as Pluronic (BASF), Synperonic (Croda) or Poloxamers (ICI) [1-7]. The CMC is sensitive to the chemical composition of the Pluronic and various grades are available. It has been well established that Pluronics form temperature sensitive micelles that adopt a core-shell morphology, where the more hydrophobic domain (PPO) forms the core and the hydrophilic domain (PEO) forms the hydrophilic corona, i.e. the shell [8-12].

In practical applications, it is usual that polymer-surfactant mixtures are employed as these often have improved properties derived by complex formulation; the addition of ionic/non-ionic surfactants to such polymeric surfactant solutions. The improvement in performance arises due to the synergistic or antagonistic interactions between the various surfactants [13]. A considerable number of studies have focused on determining the onset of the micellization process and the composition/morphology of the mixed micelles formed [9,14-16], though theoretical modelling is limited to systems that are considerably more simple than generally encountered in 'real-life' formulations [17,18] .

The interactions of small molecule surfactants with Pluronic are of relevance to their numerous pharmaceutical, domestic, technological and industrial applications. 
Mixtures of small molecules surfactant with Pluronic have previously been examined $[6,19,20]$ as has the effect of alcohols $[11,13,21]$. To our knowledge, there have been far fewer studies of the quaternary systems, Pluronic/small molecule surfactants/alcohol/water, at least in non (micro) emulsion systems.

Previously, we quantified the interaction between the homopolymer polyvinylpyrrolidone (PVP) and SDS in the presence of ethanol [13], and subsequently Pluronic P123 and sodium dodecylsulfate (SDS) in the presence of ethanol [11]. A range of techniques were employed including small-angle neutron scattering (SANS), surface tension and spectrofluorometry. Synergistic interactions between the SDS and P123 were observed, these interactions were characterised by the adsorption of the SDS into the PPO core. It was also observed that addition of ethanol to both P123 and SDS solubilized the PPO core, increasing the CMC of P123, and that reducing the dielectric constant of the solvent led to the formation of smaller micelles in both cases $[6,11,19]$.

Against this background, the present study was undertaken to quantify the effect of short (ethanol), medium (hexanol) and long (decanol) chain alcohols, ionic/non-ionic surfactants comprising dodecyl chain and different head group sizes (anionic SDS, cationic $\mathrm{C}_{12} \mathrm{TAB}$ and non-ionic Brij 35) on the micellar structure of one specific Pluronic, P123. These effects were investigated using SANS, pulsed-gradient spin-echo nuclear magnetic resonance spectroscopy (PGSE-NMR) and surface tension measurements. The aim is to quantify the interactions between the Pluronic and the small molecule and to elaborate the dependence on the presence of the added alcohol, and demonstrate how the partitioning of the alcohol will drive the interaction between the surfactants.

\section{Experimental}

\subsection{Materials}

Pluronic P123, $\mathrm{PEO}_{20}-\mathrm{PPO}_{70}-\mathrm{PEO}_{20}$, average $\mathrm{Mn}_{\mathrm{n}} 5800 \mathrm{~g} \mathrm{~mol}^{-1}$ (Aldrich) was used as received. Sodium dodecylsulfate (SDS) (Aldrich, purity 98.5\%), dodecyltrimethylammonium 
bromide (C12TAB) (Aldrich, purity 98\%), polyoxyethylene (23) lauryl ether (Brij 35) (Aldrich) were all used as received. Deuterated SDS (d-SDS) and C12TAB (d-C12TAB) for SANS experiments were purchased from Aldrich (99.9\%) and used as received. Ethanol, hexanol and decanol, all protonated (Fisher Scientific), deuterium oxide (Aldrich, 99.9\%) were used as received.

\subsection{Methods}

\subsubsection{Surface Tension}

The surface tension measurements of pure and the mixed systems were carried out using a maximum bubble pressure tensiometer (SITA science on-line t60, Germany), calibrated by reference to de-ionized water. Surface tension was recorded at a bubble life time of value $10 \mathrm{~s}$. All measurements were taken at $25 \pm 1^{\circ} \mathrm{C}$.

\subsubsection{Small-angle neutron scattering}

SANS measurements were performed at $25^{\circ} \mathrm{C}$ on the fixed-geometry, time of flight LOQ diffractometer (ISIS Spallation Neutron Source, Oxfordshire, UK). Neutron wavelengths spanning 2.2-10 $\AA$ were used to access a $Q$ range $(Q=4 \pi \sin (\theta / 2) / \lambda)$ of approximately $0.008-0.25 \AA^{-1}(25 \mathrm{~Hz})$, with a fixed sample-detector distance of $4.1 \mathrm{~m}$. The samples were contained in $1 \mathrm{~mm}$ path length, UV-spectrophotometer grade, quartz cuvettes (Hellma) and mounted in aluminium holders on top of an enclosed, computer-controlled, sample chamber. Temperature control was achieved through the use of a thermostatted circulating bath pumping fluid through the base of the sample chamber. Under these conditions a temperature stability of better than $\pm 0.5^{\circ} \mathrm{C}$ can be achieved. Experimental measuring times were approximately $40 \mathrm{~min}$.

All scattering data were normalised for the sample transmission and the incident wavelength distribution, corrected for instrumental and sample backgrounds using a quartz cell filled with $\mathrm{D}_{2} \mathrm{O}$ (this also removes the incoherent instrumental background arising from vacuum windows, etc.), and corrected for the linearity and efficiency of the detector response using the instrument specific software package. The data were put onto an absolute scale using a well characterized partially deuterated polystyrene blend standard sample. 
The intensity of the scattered radiation, $\mathrm{I}(\mathrm{Q})$, as a function of the wave vector, $\mathrm{Q}$, is given by :

$$
I(Q)=N_{m} V_{m}^{2}(\Delta \rho)^{2} P(Q) S(Q)+B_{\text {inc }}
$$

where $P(Q)$ describes the morphology of the scattering species, $S(Q)$ describes the spatial arrangement of the micelles in solution, $N_{m}$ is the number of micelles per unit volume, $V_{m}$ is the volume of the micelle, $\Delta \rho$ is the difference between the neutron scattering length density of the micelle and the solvent and $B$ inc is the incoherent background scattering.

Assuming there are three discrete regions; core, shell and the continuous solvent, the macroscopic scattering cross section is given as the particle number density multiplied by the square of the single-particle form factor, $P(Q)$ :

$$
P(Q)=N\left[\left(\rho_{A}-\rho_{C}\right) V_{A} \frac{3 j_{1}\left(Q R_{A}\right)}{Q R_{A}}+\left(\rho_{B}-\rho_{C}\right) V_{A+B}\left(\frac{3 j_{1}\left(Q R_{B}\right)}{Q R_{B}}-\frac{3 j_{1}\left(Q R_{A}\right)}{Q R_{A}}\right)\right]^{2}
$$

where $N$ is the number of core-shell particles per unit volume of solution, $\rho_{A}$ is the scattering length density for the core, $\rho_{B}$ is the scattering length density for the shell, $\rho_{c}$ is the scattering length density for the solvent, $V_{A}$ is the specific volume in the core, $V_{B}$ is the specific volume in the shell and $V_{A+B}$ is the volumes of the core and that of the shell [22].

The first part of the equation is the contribution to the differential cross section from the core with the relevant scattering length density difference that between the core and the solvent. The second part of the equation is the shell contribution $\left(R_{A}\right.$ and $\left.R_{B}\right)$ to the scattering $\left(j_{1}\right.$ is the first order spherical bessel function of the first kind) [22].

The $S(Q)$ used in the fitting routine is the rescaled mean spherical approximation provided by Hayter et al $[23,24]$ for spheres of given micellar concentration, charge 
and ionic strength, incorporating refinements for low-volume fractions and a penetrating ionic background.

The data were fitted using the SasView analysis program [25]. The software is open source and has been developed by major neutron scattering facilities; ISIS, ILL and NIST. The morphology of the micelle adopted for P123 here follows a model for that of a charged particle with core shell morphology. The shell may also contain solvent and/or the added surfactant or alcohol, so an intermediate scattering length density (SLD) between that of the core and solvent could be used. In some cases e.g., P123/small molecule surfactant mixed micelles, we invoke a slightly simpler model of a charged solid ellipsoidal micelle (i.e. no shell) as there are no signature of the shell (a bump at high Q) in the data. A detailed structure of the core-shell micelle is not required as it will be difficult to extract meaningful information without over parameterising the fit. It should be also noted that for anisotropic particles such as ellipsoids, there is a numerical integration over the particle orientation. A polydispersity parameter was included in the fitting routine and for most of the samples, it was set at 0.15 .

The mixed micelle has been treated as a two component (P123 and small molecule surfactant, SMS) surfactant system [26]. The volume of this mixed micelle having aggregation number, $N$, is calculated by:

$$
V_{m}=N\left(x_{1} v_{S M S}+\left(1-x_{1}\right) v_{P 123}\right)
$$

where $x_{1}$ is the mole fraction of SMS in the mixed micelle. $v_{S M S}$ and $v_{P 123}$ are the molecular volumes of SMS and P123 respectively. The micelle composition was extracted from the SANS data without any data fitting [6], from the ratio of the intensities of scattering $R(Q)$ obtained with h- and d-SMS at the same composition via:

$$
V f_{S M S}=\left(\frac{(\sqrt{R(Q)}-1)\left(\rho_{P 123}-\rho_{D 2 O}\right)}{\left(\rho_{h-S M S}-\rho_{P 123}\right)-\sqrt{R(Q)}\left(\rho_{d-S M S}-\rho_{P 123}\right.}\right)
$$

where 


$$
R(Q)=\frac{I(Q)^{h-S M S . P 123}-B_{i n c}^{h-S M S . P 123}}{I(Q)^{d-S M S . P 123}-B_{i n c}^{d-S M S . P 123}}
$$

For the P123-Brij 35 mixed micelles, the micelle composition was not extracted using the same method as it was not possible to obtain deuterated Brij 35.

\subsubsection{Pulsed-Gradient Spin Echo Nuclear Magnetic Resonance (PGSE-NMR)}

PGSE-NMR (diffusion NMR) experiments were carried out at $25^{\circ} \mathrm{C}$ on a $400 \mathrm{MHz}$ Bruker FT NMR spectrometer. The gradient pulse duration ( $\delta)$ was set to $1 \mathrm{~ms}$ and the magnetic field gradient $(\mathrm{G})$ was varied from 5 to $500 \mathrm{G} / \mathrm{cm}$. The diffusion time $(\Delta)$ was set to 300 ms. Further information on PGSE-NMR may be found in reference [27]. 8 scans were accumulated over 16 gradient steps. The self-diffusion coefficient was extracted by using CORE [28] .

The partitioning of the alcohol and small molecule surfactant may be easily quantified by NMR diffusion measurements since the measured self-diffusion coefficient is an average value containing contributions from the monomeric and micellized surfactant, weighted by the respective concentrations. This is equivalent to the fractional time spent by each molecule in a given environment, and is frequently expressed in terms of the fractional micelle composition, $p_{\text {micelle }}^{P 123}$ [29]:

$$
\bar{D}^{P 123}=p_{\text {micelle }}^{P 123} D_{\text {micelle }}^{P 123}+\left(1-p_{\text {micelle }}^{P 123}\right) D_{\text {monomer }}^{P 123}
$$

Where $\bar{D}^{P 123}$ is the measured self-diffusion coefficient, $D_{\text {micelle }}^{P 123}$ is the micelle selfdiffusion coefficient and $D_{\text {monomer }}^{P 123}$ is the self-diffusion coefficient of P123 monomers.

A similar analysis [29] to extract the partitioning of the alcohol $\left(p_{\text {free }}^{R-O H}\right)$ and the small molecule surfactant, may be conducted as shown in equation 6 , where $D_{\text {micelle }}$ is the measured self-diffusion coefficient, $D_{\text {micelle }}^{R-O H}$ is the micelle self-diffusion coefficient after alcohol solubilisation and $D_{\text {free }}^{R-O H}$ is the free alcohol self-diffusion coefficient. 


$$
p_{\text {free }}^{R-O H}=\frac{D_{\text {free }}^{R-O H}-D_{\text {micelle }}^{R-O H}}{D_{\text {free }}^{R-O H}-D_{\text {micelle }}}
$$

Hence from a single PGSE-NMR measurement, $p_{\text {free }}^{R-O H}$ and $p_{\text {micelle }}^{P 123}$ can be obtained.

\section{Results and Discussion:}

3.1 Onset of Micellization. Surface tension is a particularly convenient experimental technique to determine the onset of micellization. However, for complex systems e.g. mixtures of surfactants/water, the discontinuities in the surface tension may be at least initially counterintuitive. For example, when one dilutes a binary surfactant/water system through its $\mathrm{CMC}$, does the experimental design maintain a fixed ratio of two surfactants or a fixed concentration of one of the materials. The surface tension curves would be quite different, as the system would evolve from mixed micelles to none or mixed to pure. This is especially important when there is a hydrophobic component present. It also should be noted that surface tension is a measure of the surface properties, hence, the difficulty of designing an experiment investigating bulk characteristics and competitive interactions. Accordingly, selections of behaviours are presented in Figure 1.

Figure 1, compiles several approaches we have used to design surface tension experiments. Focusing on the SDS case, which is more interesting, if we dilute P123 in simple aqueous solvent (open circles), there is clearly one break point in the curve yielding $\mathrm{CMC} \sim 0.1 \mathrm{wt} \%$. In the presence of SDS, keeping [P123] $=0.5 \mathrm{wt} \%$ (filled grey circles), several behaviours that are insensitive to the ethanol presence can be noticed. Three different SDS concentration regions can be distinguished, for low SDS concentrations, up to $1 \mathrm{mM}$, the surface tension remains unaffected by the SDS and remains dominantly controlled by the P123; over an intermediate SDS concentration, up to $10 \mathrm{mM}$, the surface tension increases sharply to a plateau, upon further increase of SDS concentration, the surface tension again decreases. 
As outlined in Table 1a, the CMC of pure SDS (empty squares) is $7.9 \mathrm{mM}$ which agrees well with earlier reported data [30-32]. In the presence of $0.5 \mathrm{wt} \%$ of the polymer, the CMC of SDS is lowered significantly from $7.9 \mathrm{mM}$ to $1.2 \mathrm{mM}$, such decrease in the CMC could be understood as the aggregation of the SDS on the polymer.

In the presence of ethanol, the CMC and the surface tension of SDS (filled squares) is also remarkably reduced, confirming that the micellization is promoted by the presence of ethanol- the cosurfactant effect. In the presence of $10 \mathrm{wt} \%$ ethanol, the P123 surface tension curve (filled circles), shows one break in the curve yielding a $\mathrm{CMC}$ of $\sim 0.6 \mathrm{wt} \%$, which in comparison to the CMC of the simpler P123 solution, $\sim 0.1$ $\mathrm{wt} \%$, confirms that the micellization process is unfavoured (the CMC increases) as the solvent is less hydrophobic.

Table 1a: Surface tension derived CMC/critical aggregate concentration (CAC) values as a function of P123, SDS and ethanol concentration in water

\begin{tabular}{|c|c|c|c|}
\hline System & $\mathrm{CMC}$ or $\mathrm{CAC}$ & System & $\mathrm{CMC}$ or $\mathrm{CAC}$ \\
\hline SDS/water & $7.9 \mathrm{mM}$ & $\mathrm{C}_{12} \mathrm{TAB} /$ water & $15 \mathrm{mM}$ \\
\hline SDS/0.5wt\% P123-water & $1.2 \mathrm{mM}$ & $\mathrm{C}_{12} \mathrm{TAB} / 0.5 \mathrm{wt} \%$ P123-water & $3.7 \mathrm{mM}$ \\
\hline SDS/10wt $\%$ ethanol & $4.5 \mathrm{mM}$ & $\mathrm{C}_{12} \mathrm{TAB} / 10 \mathrm{wt} \%$ ethanol & $12 \mathrm{mM}$ \\
\hline P123/water & $0.1 \mathrm{wt} \%$ & P123/water & $0.1 \mathrm{wt} \%$ \\
\hline P123/10 wt $\%$ ethanol & $0.6 \mathrm{wt} \%$ & $\mathrm{P} 123 / 10 \mathrm{wt} \%$ ethanol & $0.6 \mathrm{wt} \%$ \\
\hline P123/50 mM SDS-water & $0.3 w t \%$ & P123/50 mM C12TAB-water & $0.04 w t \%$ \\
\hline P123/50 mM SDS- $10 w t \%$ ethanol & $0.6 \mathrm{wt} \%$ & P123/50 mM C C12 TAB- $10 \mathrm{wt} \%$ ethanol & $0.1 \mathrm{wt} \%$ \\
\hline
\end{tabular}

Table 1b: Surface tension derived CMC/critical aggregate concentration (CAC) values as a function of P123, $C_{12} \mathrm{TAB}$ and ethanol concentration in water

When a fixed concentration of SDS /ethanol is kept (open diamonds), a decrease in surface tension is observed by increasing the P123 concentration as the less surface active species (small molecule surfactant and ethanol) are being stripped from the surface and it is being replaced with more active ones, P123. While if we keep a fixed SDS/hexanol concentration, the surface tension increases by increasing the P123 concentration as it has a lower surface activity compared to the SDS/hexanol and water mixture (Supplementary material). 


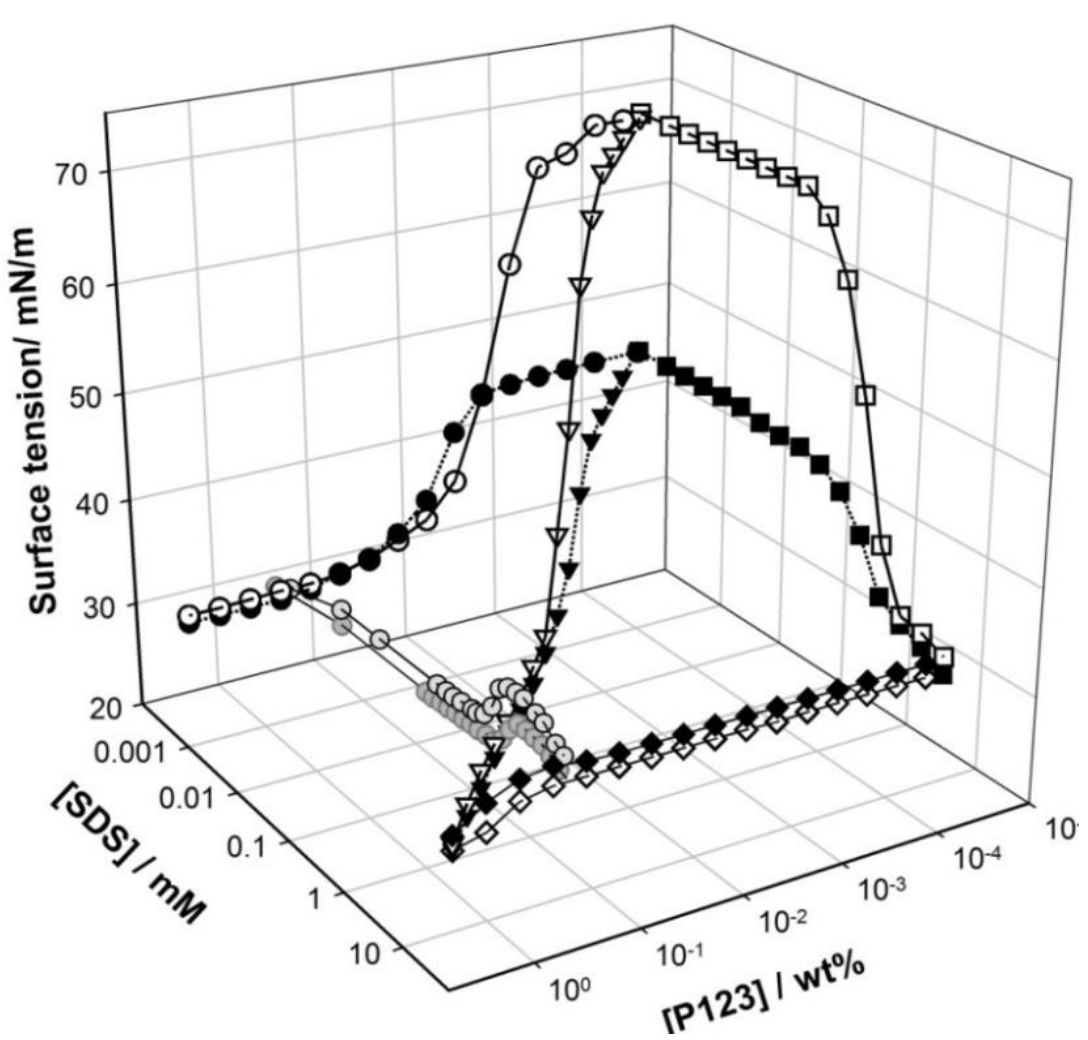

Figure 1: Surface tension as a function of surfactant concentration: open squares, $\square=$ [SDS/water], filled squares, $\boldsymbol{\square}=[$ SDS+constant ethanol concentration , $10 \mathrm{wt} \%$ in water], open diamonds, $\diamond=[\mathrm{P} 123+$ constant SDS and ethanol concentration, $50 \mathrm{mM} \& 10 \mathrm{wt} \%$ in water], filled diamonds, $=[P 123+$ constant SDS concentration, $50 \mathrm{mM}$ in water] ,open triangles, $\triangle=$ [P123+SDS in water], filled triangles, $\Delta=[\mathrm{P} 123+\mathrm{SDS}+$ constant ethanol concentration, $10 \mathrm{wt} \%$ in water], grey open circles across the SDS axis, $\bigcirc=[$ SDS+constant P123 concentration, $0.5 \mathrm{wt} \%$ in water]/grey filled circles across the SDS axis, $\bigcirc=$ [SDS+constant P123 and ethanol concentration, $0.5 \mathrm{wt} \%$ \& $10 \mathrm{wt} \%$ ethanol in water], open circles, $\bigcirc=[\mathrm{P} 123 /$ water], filled circles , $=[\mathrm{P} 123+$ constant ethanol concentration, $10 \mathrm{wt} \%$ in water $]$

Changes in the system along the concentration range of ternary mixtures of P123 or small molecule surfactant and the hydrophobic alcohols (hexanol and decanol) in water, where the alcohol is kept constant, are quite significant. Below the $\mathrm{CMC}$, where surfactant monomers only are present, the alcohol solubilisation is lower and the alcohol phase separates out of the solution. This phase behaviour and the maximum solubilisation of the surfactant were the key to the alcohol concentration selection in the P123 in water and P123-small molecule surfactant mixtures.

The interactions contributing to the formation of the mixed micelles can be described as synergistic or antagonistic in terms of the change in the CMC. We use the terms in a more general sense. In these quaternary systems investigated, interactions with varying strengths between the surfactants were observed for SDS-P123, C12TAB- 
P123 and Brij 35-P123 in water and water/alcohol mixtures. These interactions are observed as the small surfactant molecules are being included in between the Pluronic molecules. The size and shape of the self- assembled structure is dictated by these interactions. SANS has been used to quantify these structures, and again illustrative data are presented.

3.2 Effect of Alcohols on the Pluronic Micelle Structure. Scattering profiles for $5 \mathrm{wt} \%$ Pluronic P123 above the CMC are shown in Figure 2. The scattering profile of $5 \mathrm{wt} \%$ P123 in $\mathrm{D}_{2} \mathrm{O}$ yields considerable scattering intensity with a noticeable "bump" around $\mathrm{Q}=0.09 \AA^{-1}$, reflecting a sharp discontinuity in the scattering length density profile across the micelle indicative of the well-defined core and corona regions. Fitting the data to the core-shell model described earlier shows that the P123 micelles are slightly polydisperse with a spherical core of $57 \AA \pm 1$ and corona $14 \AA \pm 0.5$ with the latter adopting Gaussian statistics [33].

Given that the CMC of P123 increases in the presence of ethanol, it is more likely that this decreasing intensity at low $\mathrm{Q}$ after the addition of $10 \mathrm{wt} \%$ ethanol, Figure 2, is due to the reduction of the number and/or the volume of the micelle and/or a change in the interaction between the micelles via the structure factor. This could be simply attributed to the fact that the ethanol/water mixture solubilises the hydrophobic PPO core more than pure water [34,35], changing the effective HLB of the surfactant. Support (albeit indirect) arises from an interpretation of the micellar volume fraction parameter extracted from the Hayter-Penfold fit, in that all attempts to describe the data with a volume fraction greater than 0.05 (in essence, the mass fractions of the Pluronic plus the solubilised alcohol) led to poor fits. It was concluded therefore, that the ethanol displaces the water in the EO-rich shell, thereby not significantly increasing the fraction of the micelle bounded material. 
By contrast, in the case of $1 \mathrm{wt} \%$ hexanol and $0.1 \mathrm{wt} \%$ decanol, the scattering intensity at low $\mathrm{Q}$ increases reflecting the presence of more and/or larger micelles as the hydrophobic alcohol is incorporated into the micellar core. Making the reasonable assumption that the hydroxyl group is present at the core/corona interface to maintain its hydration, the modelling shows that this drives the formation of a larger micelle. The slight shift of the structure factor, extracted from the fit (inset in Figure 2) towards higher $\mathrm{Q}$ values is indicative of the decreasing distance between the micelles associated with the increase in the micelle size. The key parameters, extracted from the fitting, are listed in Table 2.

The SLD for the solvent $\left(\mathrm{D}_{2} \mathrm{O}\right)$ was kept constant in the fitting routine for most of the samples at $6.39 \times 10^{-6} \AA^{-2}$, but was adjusted accordingly using the partitioning values extracted from PGSE-NMR data analysis. For example, the samples containing $10 \mathrm{wt} \%$ ethanol, where PGSE-NMR has shown that $2.5 \%$ only remains unbound in the solvent, the SLD of the solvent $\left(\mathrm{D}_{2} \mathrm{O}\right.$ and some fraction of the h-ethanol) and the core (PPO and the remaining fraction of h-ethanol) were included in the fitting routine based on some simple assumptions; (i) all the ethanol partitions into the core, (ii) all the ethanol partitions into the shell, (iii) some distribution of the ethanol between the core and the shell. For each simulation, the SLD of the appropriate region was estimated based on the composition, and this value is used as a input parameter ("guess") or occasionally held constant in the fitting routine. Based on this analysis, we conclude that the majority of the ethanol is located in the shell of the micelle. PGSE-NMR has also shown that both hexanol and decanol partition into the micelle, but in these cases, due to their low concentration, the change in the solvent SLD was negligible.

Figure 2 also shows the effect of hexanol on the aggregation behaviour of the P123 micelle. Addition of $1 \mathrm{wt} \%$ hexanol resulted in a large increase in the scattering intensity at low $\mathrm{Q}$. The linearity observed in the low $\mathrm{Q}$ region is suggestive of the ellipsoidal micelles [36,37]. Data fitting suggests the formation of prolate ellipsoidal 
micelles with major axis of $160 \AA$ and a minor axis of $55 \AA$ A. Key fitting parameters are listed in Table 2.

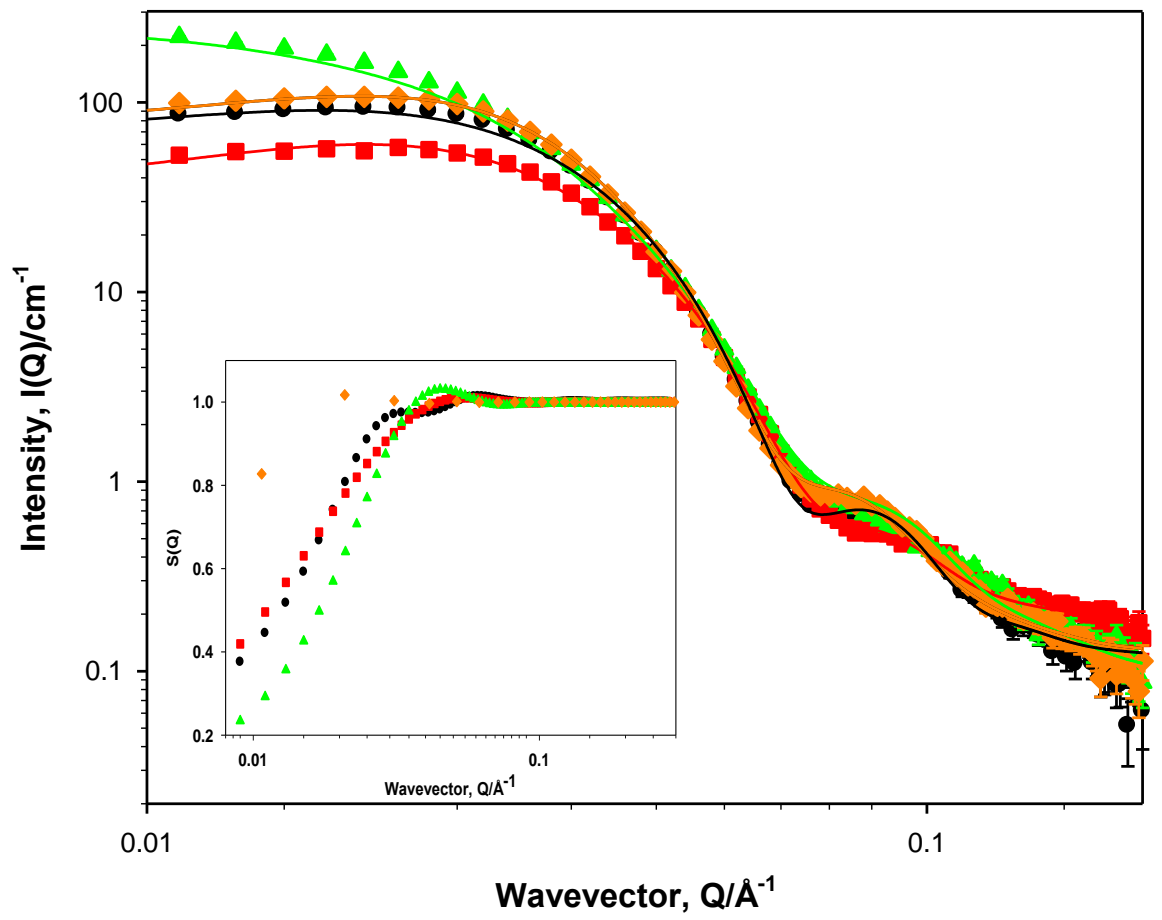

Figure 2: SANS from $5 \mathrm{wt} \% \mathrm{P} 123$ solutions as a function of alcohol concentration; black circles, [alcohol] $=0 \mathrm{wt} \%$, red squares, $\square,[$ ethanol] $=10 \mathrm{wt} \%$, green triangles, $\boldsymbol{\Delta}$,[hexanol] $=1 \mathrm{wt} \%$ and orange diamonds,,$[$ decanol $]=0.1 \mathrm{wt} \%$. The inset shows the structure factor $S(Q)$ extracted from the fit of the same data in the same colouring order of the main graph. Solid lines are fits to the core-shell model, see Table 2.

The material-balance equations, described by Slawecki et al [38] allow us to quantify the composition of the core and shell of the P123 micelle in $\mathrm{D}_{2} \mathrm{O}$ using the SANS data. There were 5-6 $\mathrm{D}_{2} \mathrm{O}$ molecules per EO monomer in the shell. The aggregation number, $110 \pm 5$, is in good agreement with other reported values from data previously fitted to the Pedersen model for Pluronics in solution [39] at the same polymer concentration and temperature, $5 \mathrm{wt} \%[11,34]$. Percentage of EO monomers inside the PO core was found to be $2.96 \%$ which agrees with the simple understanding of a core shell model, where both regions are well defined.

Self-diffusion coefficients obtained by PGSE-NMR were used to determine values of $p_{\text {free }}^{O H}$ (free fraction of alcohol not solubilised the micelles) as shown in equation (6). Applying the values obtained for $p_{\text {free }}^{O H}$ to the mass balance equation, one may correct to a first assumption that $50 \%, 15 \%$ and $3 \%$ of these micelle volumes are the 
solubilized alcohols, giving an estimate of the aggregation number of 60, 200 and 160 for micelles with ethanol, hexanol and decanol respectively. These estimates of the aggregation numbers are entirely consistent with the measured P123 diffusion rates.

Table 2: Fitting parameters for $5 \mathrm{wt} \% \mathrm{P} 123$ as a function of alcohol concentration at $25^{\circ} \mathrm{C}$ (from SANS and PGSE-NMR)

\begin{tabular}{|c|c|c|c|c|}
\hline Alcohol concentration (wt $\%)$ & $0 w t \%$ & $10 \mathrm{wt} \%$ Ethanol & 1wt $\%$ Hexanol & $0.1 \mathrm{wt} \%$ Decanol \\
\hline Core radius $( \pm 1, \AA)$ & 57 & 50 & a: $160 / b: 55$ & 60 \\
\hline Shell thickness $( \pm 0.5, \AA)$ & 14 & 13.5 & -- & 13 \\
\hline Volume fraction of hard spheres $( \pm 0.001)$ & 0.05 & 0.05 & 0.05 & 0.05 \\
\hline Volume of the micelles $\left(\mathrm{nm}^{3}\right), V_{m i c}$ & 1500 & 1100 & 2100 & 1600 \\
\hline Free alcohol fraction, $p_{\text {free }}^{O H}$, from NMR & -- & 0.25 & 0.03 & 0 \\
\hline Aggregation number, $\mathrm{N}_{\text {agg }}( \pm 5)$, & 110 & $60^{\mathrm{a}}$ & 200 & 160 \\
\hline Micelles number density, $n, 10^{16} \mathrm{~cm}^{-3}$ & $3.5^{\mathrm{b}}$ & 4.7 & 2.0 & 3.2 \\
\hline
\end{tabular}

Significant changes in micelle morphology are observed when small molecule surfactants are added to the P123/alcohol systems. Take for example the SDS case, on addition of $50 \mathrm{mM}$ SDS to the $5 \mathrm{wt} \%$ P123 aqueous and alcohol/aqueous solutions, Figure 3, SANS data show significant loss in the scattering intensity and the emergence of correlation peaks at higher $Q$ values which together indicates the formation of smaller, mixed, charged micelles.

Adding the SDS has resulted in the loss of the "bump" at $\sim 0.09 \AA^{-1}$. The addition of the ethanol to the P123/SDS mixed micelle led to a slight decrease in the scattering intensity with no significant change in the position of the correlation peak. As shown previously in Figure 2, the hydrophobicity of the micellar core increases by increasing the hydrophobicity of the alcohols, evident by the increase in the scattering intensity, along with a shift in the correlation peak toward the lower $\mathrm{Q}$ region indicating the formation of larger aggregates, Table 3. The scattering from these mixed micelles were fitted using a form factor describing the micelle as an oblate ellipsoid with a charged structure factor as calculated by Hayter and Penfold. The key parameters are listed in Table 3. 
It is widely accepted that SDS absorbs into the PPO core as the interaction between SDS and the hydrophobic PPO block of P123 is stronger than that between SDS and the hydrophilic PEO block where it renders the core less hydrophobic [15,16]. Jansson et al [42] showed that at low SDS/P123 mole ratios, the principle structure is a P123 micelle with co-micellised SDS. At higher SDS/P123 mole ratios, P123 micelles are broken up, forming SDS-rich micelles co-micellised with few P123 molecules.

Table 3: SANS parameters for $5 w t \%$ P123- $50 \mathrm{mM}$ h-SDS as a function of alcohol concentration

\begin{tabular}{lcccc}
\hline Alcohol concentration $(\mathrm{wt} \% / \mathrm{v})$ & $0 \mathrm{wt} \%$ & $10 \mathrm{wt} \%$ Ethanol & $1 \mathrm{wt} \%$ Hexanol & $0.1 \mathrm{wt} \%$ Decanol \\
\hline Radius A, polar $( \pm 0.5, \AA)$ & 17 & 17 & 21 & 19 \\
Radius B, equatorial $( \pm 1, \AA)$ & 39 & 37 & 46 & 39 \\
Volume fraction of hard spheres $( \pm 0.001)$ & 0.06 & 0.05 & 0.06 & 0.06 \\
Volume of the micelles $\left(\mathrm{nm}^{3}\right)$ & 110 & 95 & 185 & 120 \\
Mixed micelle aggregation number $( \pm 5)$ & 12 & 10 & 20 & 13 \\
Micelles number density, $n, 10^{17} \mathrm{~cm}^{-3}$ & 5.9 & 5.2 & 3.2 & 5.0 \\
\hline
\end{tabular}

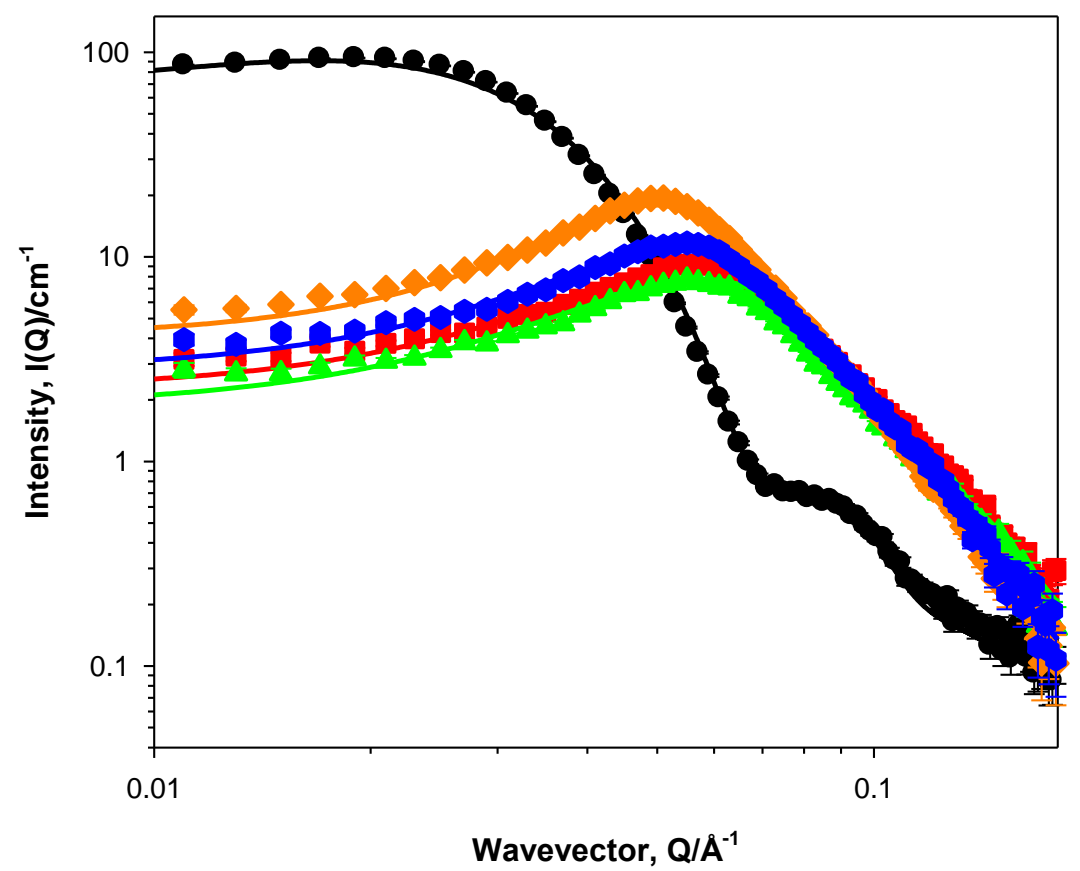

Figure 3: SANS from $5 \mathrm{wt} \%$ P123 solutions+ $50 \mathrm{mM}$ h-SDS as a function of alcohol chain length; grey circles,, , [alcohol] $=0 \mathrm{wt} \%$ and $[\mathrm{h}-\mathrm{SDS}]=0 \mathrm{mM}$, red squares, $\square$,[alcohol] $=0 \mathrm{wt} \%$, green triangles, $\mathbf{\Delta}$, [ethanol] $=10 \mathrm{wt} \%$, orange diamonds, $\bullet$, [hexanol] $=1 \mathrm{wt} \%$ and blue hexagons,, [decanol] $=0.1 \mathrm{wt} \%$. Solid lines correspond to model fits as discussed in the text. Data above $Q=0.15 \AA^{-1}$ have been omitted for clarity. 
One could envisage a situation where the bulkiness of the head group of the small molecule or its charge density would control the size and the hydration level of the mixed micelle, Table 4 . SANS data, Figure 4 shows the effect of the head group size on the scattering intensity. The mixed micelle aggregation number shows variations with changing the small molecule surfactant charge. For the P123/SDS system, the aggregation number decreases after adding SDS to the block copolymer (Nagg = $12 \pm$ 5) $[11,15,16,43]$, which is expected as the head group $\left(\mathrm{SO}_{4} \mathrm{Na}^{+}\right)$is forming small curved surfaces, whereas in the $\mathrm{P} 123 / \mathrm{C}_{12} \mathrm{TAB}$ system, the $\mathrm{C}_{12} \mathrm{TAB}\left(\mathrm{N}\left(\mathrm{CH}_{3}\right)_{3} \mathrm{Br}-\right)$ is likely to be less charged where the degree of counter-ion dissociation is less, hence the decrease in the curvature and the formation of larger structures (Nagg= $20 \pm 5)$. Upon introducing a non-ionic surfactant, Brij 35, there is a further decrease in the charge which forms even bigger structures $(\mathrm{Nagg}=25 \pm 5)$.

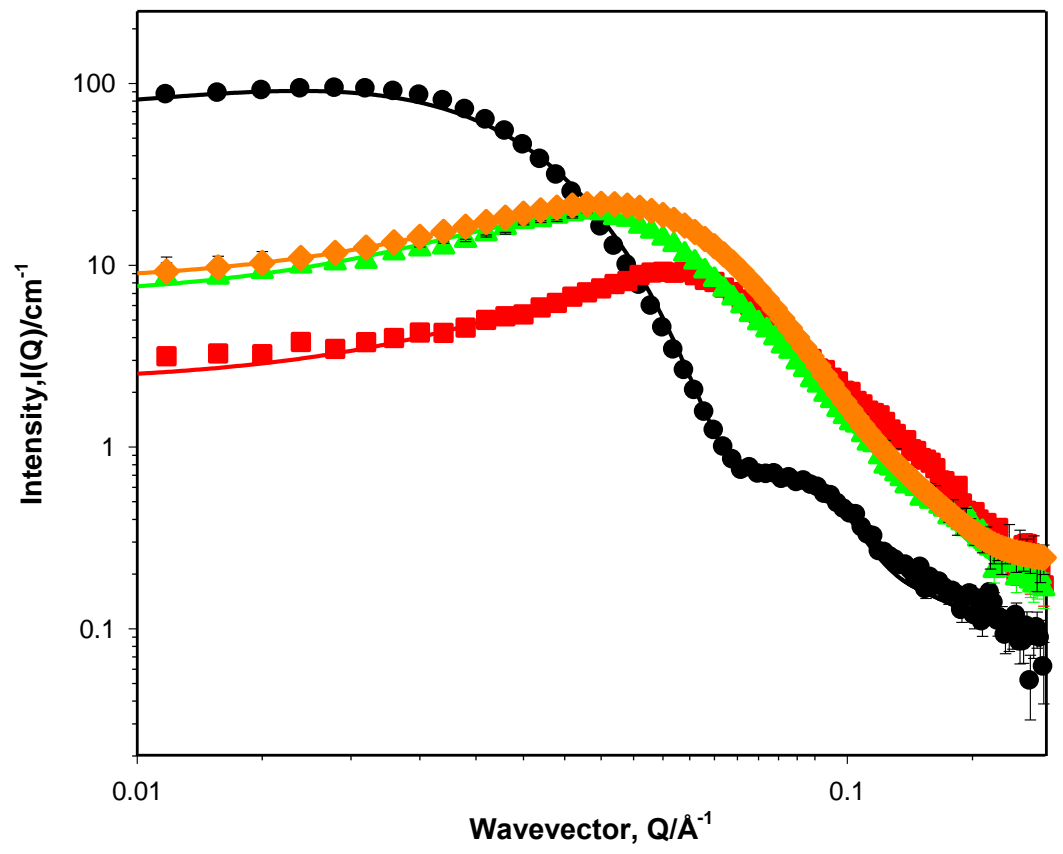

Figure 4: SANS from $5 \mathrm{wt} \%$ P123 solutions as a function of small molecule surfactant head group size; black circles, $\mathbf{~ , ~ [ s u r f a c t a n t ] ~}=0 \mathrm{mM}$, red squares, $\mathbf{a},[\mathrm{h}-\mathrm{SDS}]=50 \mathrm{mM}$, green triangles, $\Delta,\left[\mathrm{h}-\mathrm{C}_{12} \mathrm{TAB}\right]=$ $50 \mathrm{mM}$, orange diamonds,,$[$ Brij 35]= $50 \mathrm{mM}$ 
Table 4: SANS parameters for $5 \mathrm{wt} \% \mathrm{P} 123$ as a function of $50 \mathrm{mM}$ h-surfactants in $\mathrm{D}_{2} \mathrm{O}$

\begin{tabular}{llcc}
\hline Surfactant & SDS & $\mathrm{C}_{12}$ TAB & Brij 35 \\
\hline Radius A, polar $( \pm 0.5, \AA$ A $)$ & 17 & 23 & 26 \\
Radius B, equatorial $( \pm 1, \AA)$ & 39 & 45 & 45 \\
Volume fraction of hard spheres $( \pm 0.001)$ & 0.06 & 0.06 & 0.09 \\
Volume of the micelles $\left.(\mathrm{nm})^{3}\right)$ & 110 & 195 & 210 \\
Mixed micelle aggregation number, \pm 5 & 12 & 20 & 25 \\
Micelles number density $, n, 10^{17} \mathrm{~cm}^{-3}$ & 5.9 & 3.1 & 4.3 \\
\hline
\end{tabular}

Figure 5 shows the variations in the scattering data at lower P123 and small molecule surfactant concentrations but at identical P123/surfactant ratio and above

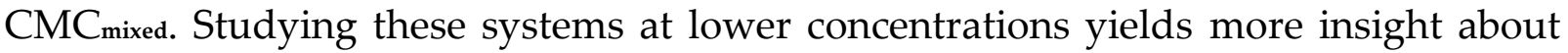
the shape of the aggregates formed and the nature of the interactions between the surfactants. At low SDS concentrations, $5 \mathrm{mM}$, there is significantly less scattering intensity indicating the presence of small, charged micelles as a result of the strong adsorption of the SDS into the PPO core and the synergistic interaction between SDS and P123. On increasing the head group size of the surfactant, i.e. C12TAB, the scattering intensity is recovered where the micelle also regains its core-shell morphology demonstrating antagonistic interactions between both surfactants. Upon a further increase in the head group size; Brij 35, the scattering pattern adopts a slope of -2 at high $Q$ confirming the formation of a mixed oblate elliptical micelle. The synergistic interactions between P123 and Brij 35 as reported in earlier reports [44,45], occurs as both surfactants have hydrated EO chains when in contact with water, which results in a full miscibility of both surfactants in the mixed micelle. The antagonistic interactions between $\mathrm{P} 123$ and $\mathrm{C}_{12} \mathrm{TAB}$ could be related to two possible explanations. The first being the large size of the head group where it shields the hydrophobic core, and suppress the presence of the polymer at the hydrocarbonwater interface. The second explanation is related to the electrostatic repulsion as a result of a slight positive charge present on the polymer, originating from the protonation of the ether oxygen in the PEO chains $[46,47]$. 


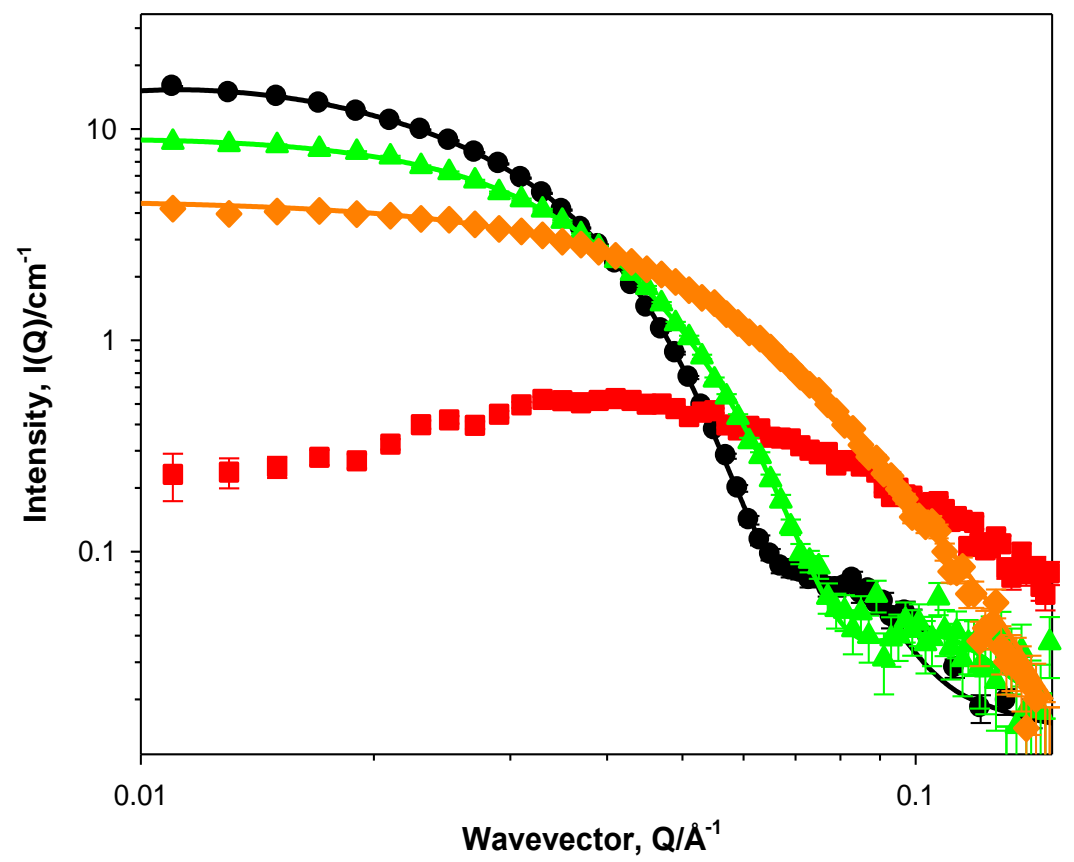

Figure 5: SANS from $0.5 \mathrm{wt} \% \mathrm{P} 123$ solutions as a function small molecule surfactant head group size; black circles, $\mathbf{O}$, [surfactant] $=0 \mathrm{mM}$, red squares,,$[\mathrm{h}-\mathrm{SDS}]=5 \mathrm{mM}$, green triangles, $\Delta,\left[\mathrm{h}-\mathrm{C}_{12} \mathrm{TAB}\right]=5$ $\mathrm{mM}$, orange diamonds,,$[$ Brij 35$]=5 \mathrm{mM}$

Table 5: SANS parameters for $0.5 \mathrm{wt} \% \mathrm{P} 123$ as a function of $5 \mathrm{mM}$ h-surfactants in $\mathrm{D}_{2} \mathrm{O}$

\begin{tabular}{lccc}
\hline Surfactant & No SMS added & C12TAB & Brij 35 \\
\hline Radius A $( \pm 1, \AA)$ & 57 & 42 & 23 \\
Thickness/Radius B $( \pm 1, \AA)$ & 17 & 12 & 51 \\
Volume fraction of hard spheres $( \pm 0.001)$ & 0.005 & 0.005 & 0.01 \\
Volume of the micelles $\left(\mathrm{nm}^{3}\right)$ & 1500 & 860 & 250 \\
Micelles number density, $n, 10^{15} \mathrm{~cm}^{-3}$ & 3.3 & 5.8 & 40 \\
Mixed micelle aggregation number $( \pm 5)$ & 140 & 88 & 26 \\
\hline
\end{tabular}

3.3 Determination of the Micelle Composition. Contrast variation experiments have also been used to separate the scattering from the P123 and the small molecule surfactants; the use of d-surfactants in $\mathrm{D}_{2} \mathrm{O}$ renders them invisible. In the case of h$\mathrm{P} 123 / \mathrm{d}-\mathrm{SDS} / \mathrm{D}_{2} \mathrm{O}$, Figure $6 \mathrm{a}$, SDS is invisible and the scattering intensity observed arises predominantly from $\mathrm{P} 123$. In the h-P123/h-SDS/ $\mathrm{D}_{2} \mathrm{O}$ case, both surfactants are visible and hence the stronger scattering intensity observed. h-P123/h-C12TAB/D2O system, Figure $6 \mathrm{~b}$, shows a higher intensity profiles than the h-SDS case and a signature of a structure adopting core-shell morphology.

A crude estimate of the SDS and $\mathrm{C}_{12} \mathrm{TAB}$ fraction $\left(V f_{S M S}\right)$ presented as $\alpha$, within the aggregate could be extracted from the SANS measurements without any data fitting from the ratio of the scattering intensities, $R(Q)$, obtained with $h$ and $d$ - 
surfactants at the same composition, equation (4). For 0.5wt\% P123-5 mM SDS, $\alpha$ (SDS) $=31( \pm 5) \%[11]$, and for $0.5 \mathrm{wt} \%$ P123-5 mM C12 TAB,$\alpha\left(\mathrm{C}_{12} \mathrm{TAB}\right)=11( \pm 5) \%$.

These findings agree with the data extracted from the fitting where SDS had the strongest interaction with P123 as discussed earlier, and it forms up to $30 \%$ of the mixed micelle structure, where in the weakest interaction case (P123- $\left.\mathrm{C}_{12} \mathrm{TAB}\right)$, the small molecule surfactant makes up $11 \%$ only of the mixed micelle structure.

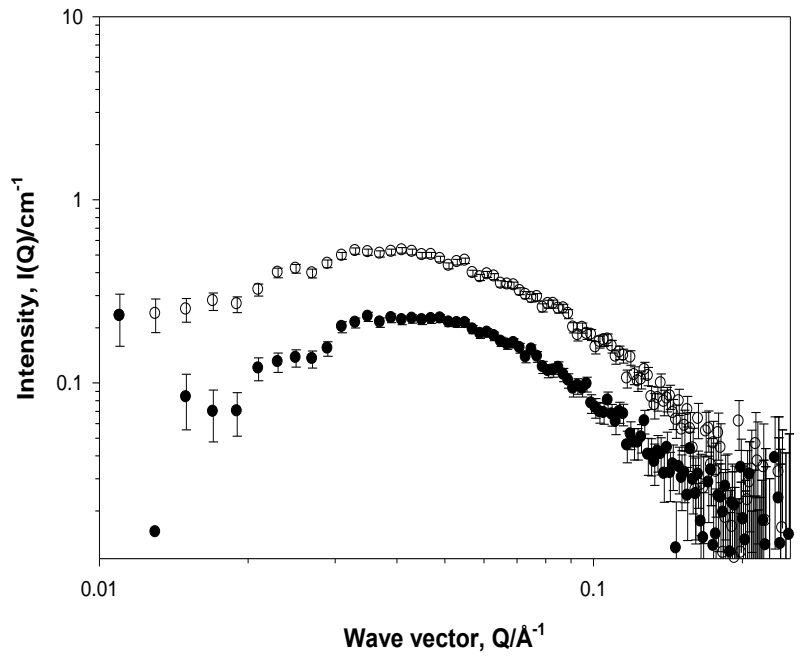

Figure 6a: SANS from $0.5 \mathrm{wt} \% \mathrm{P} 123$ and $[5 \mathrm{mM}$ h-SDS $]=$ empty circles(O), [5 $\mathrm{mM}$ d-SDS $]=$ filled circles $(\mathrm{O})$ in $\mathrm{D}_{2} \mathrm{O}$

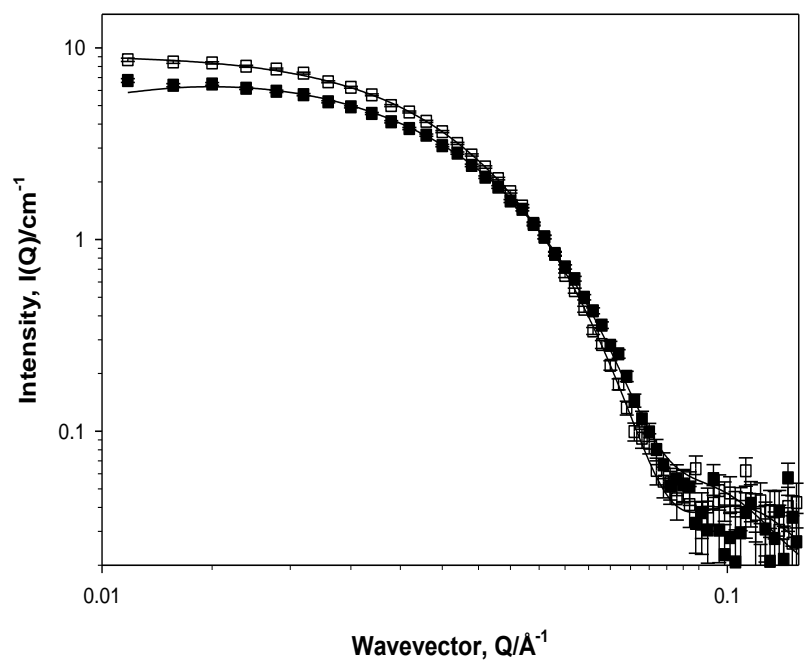

Figure 6b: SANS from $0.5 \mathrm{wt} \% \mathrm{P} 123$ and [5 $\mathrm{mM}$ h-C12TAB] = empty squares( $\square)$, [5 mM d$\left.\mathrm{C}_{12} \mathrm{TAB}\right]=$ filled squares $(\boldsymbol{)})$ in $\mathrm{D}_{2} \mathrm{O}$

Drawing the same analysis to these systems in the presence of alcohols, provides a further understanding to the role of the solvent partitioning in controlling the interactions, Table 5. When $1 \mathrm{wt} \%$ ethanol is added to the mixed system of $\mathrm{C}_{12} \mathrm{TAB}$ and P123, the subtle effect of ethanol becomes greater, yielding a higher $\mathrm{C}_{12} \mathrm{TAB}$ fraction $\left(\alpha\left(\mathrm{C}_{12} \mathrm{TAB}\right)=35( \pm 5) \%\right)$ in comparison to the $\mathrm{P} 123+\mathrm{C} 12 \mathrm{TAB}$ in $\mathrm{D}_{2} \mathrm{O}$ case, $\alpha\left(\mathrm{C}_{12} \mathrm{TAB}\right)=11( \pm 5) \%$.

Adding ethanol with the same concentration to the P123+SDS system, $(\alpha(\mathrm{SDS})=$ $28( \pm 5) \%)$, the P123+SDS effect wins over the effect of the ethanol, without showing any significant changes in the SDS fraction.

The P123+SDS effect still wins over the further increase in the hydrophobicity of the alcohol, as the fraction of SDS does not change significantly. However, the 
alcohols effect still wins over the P123+C $12 \mathrm{TAB}$ effect, where the fraction of $\mathrm{C}_{12} \mathrm{TAB}$ shows a noticeable change, Table 6.

Table 6: Mixed micelle composition in terms of small molecule surfactant fraction, $\alpha( \pm 5) \%$, as a function of alcohol concentration

\begin{tabular}{lcccc}
\hline System description & 0wt $\%$ alcohol & $1 \mathrm{wt} \%$ ethanol & $0.1 \mathrm{wt} \%$ hexanol & $0.01 \mathrm{wt} \%$ decanol \\
\hline $0.5 \mathrm{wt} \%$ P123/5 mM SDS & 31 & 28 & 30 & 31 \\
$0.5 \mathrm{wt} \%$ P123/5 mM C12TAB & 11 & 35 & 21 & 14 \\
\hline
\end{tabular}

Nagarajan and Blankschtein et al(Ref) previously stated that the free energy of formation for mixed micelles has a number of contributing terms - the deformation of the surfactant tail as it conforms to packing requirements within the micellar core, the energy of forming the aggregate core/solvent interface which depends on the aggregation number , and, finally, two terms that account for the headgroup interactions over the micellar surface, one steric and for ionic surfactants, an electrostatic interaction between the surfactant headgroups.

Making the assumption that the interfacial tension of the aggregate core/continuous phase follows the same trend as the surface tension of the bulk solution, the energy required to produce the interface decreases (favours micelle formation). The term which considers the ionic headgroup interactions is a complex one, which cannot be estimated simply. A number of factors come into play in determining the energies of the headgroup interactions, including the dielectric constant, Debye length, and radius of the micelle. For instance, the dielectric constant of the solution decreases in the presence of ethanol, which in turn influences the various charge effects such as counterion dissociation and hence the size and shape of the micelle.

For the higher alcohols, the precise mechanism is not entirely clear; one could imagine changes in the level of hydration of the ethylene oxide (EO) groups, changes in counterion binding induced due to the prevailing dielectric constant or how the composition of the micelle/solvent interface defines the interfacial tension. 


\section{Conclusions}

The effect of small molecule surfactants and alcohols on the aggregation behaviour of Pluronic P123 has been widely studied due to their extensive use in industry [1-7]. Aqueous/alcohol mixtures comprising the polymeric (Pluronic) surfactant P123, anionic SDS, cationic $\mathrm{C}_{12} \mathrm{TAB}$ and non-ionic Brij 35 have been characterised by using tensiometry, PGSE-NMR and SANS. The results are presented firstly in terms of the ternary systems, (i) Interaction between P123 in the presence of alcohols; (ii) Interaction P123 in the presence of surfactants and (iii) The quaternary system.

\subsection{Interaction between P123 in the presence of alcohols}

The partitioning in the micelle has been quantified by PGSE-NMR where it also shows that larger aggregates with slow diffusion rates were formed after adding hexanol and decanol and faster diffusing aggregates were formed after the addition of ethanol. The partitioning is in good agreement with the literature values $[37,40]$.

The micelle size decreases with the addition of ethanol, but increases with both hexanol and decanol. The micelle shape is spherical in the absence of any alcohol, and interestingly, remains so in the presence of ethanol and decanol, but forms prolate micelles in the presence of hexanol. We suggest this is a balance between the site and degree of alcohol solubilisation - ethanol is located in the outer shell of the Pluronic micelle, the more hydrophobic hexanol and decanol are solubilised into the core.

\subsection{Interaction between P123 in the presence of surfactants}

The scattering data clearly report on micelle disruption by the small molecule surfactants. By comparing the relative changes in the scattering profiles, we found that SDS has the strongest interaction with P123 [11,42], followed by Brij 35 and then $\mathrm{C}_{12} \mathrm{TAB}$ which showed the weakest interaction $[46,47]$.

\subsection{Interaction between P123 in the presence of both alcohols and surfactants}


The addition of the alcohols to the Pluronic/small molecule surfactant solution has introduced further changes to the mixed micelle composition. The effects of the alcohol have been compared with the effect of mixing the pure surfactants, in terms of the micelle composition. For (the dilute) Pluronic/SDS cases, adding the different alcohols has little effect on the micelle composition, suggesting that there is a strong interaction between SDS and P123. For systems comprising P123 and C12TAB, the $\mathrm{C}_{12} \mathrm{TAB}$ micelle mole fraction increases for all cases of alcohol, indicative of a weaker interaction between the small molecule surfactant and the Pluronic (consistent with the relative changes in $\mathrm{CMC}$ ).

This work has allowed us to extend our understanding of the interactions between different surfactants and will direct the design of further experiments where more complex systems will be investigated.

\section{Acknowledgment}

The authors thank the UK Science and Technology facilities council (STFC) for allocation of beamtime at ISIS. This work benefited from SasView software, originally developed by the DANSE project under NSF award DMR-0520547

\section{Supplementary information}

Supplementary data associated with this article can be found in the online version

\section{References}

[1] R. Kaur, S. Kumar, V.K. Aswal, R.K. Mahajan, Langmuir 29 (2013) 1182111833. DOI: $10.1021 / 1 a 401864 p$

[2] C. Booth, D. Attwood, Macromol. Rapid Commun. 527 (2000) 501-527. DOI: 10.1002/1521-3927(20000601)21:9<501::AID-MARC501>3.0.CO;2-R 
[3] P. Alexandridis, J.F. Holzwarthf, T.A. Hatton, Macromolecules 27 (1994) 24142425. DOI: 10.1016/j.jcis.2012.09.028

[4] G. Marinov, B. Michels, R. Zana, Langmuir 7463 (1998) 2639-2644. DOI: 10.1021/la971069w

[5] P. Alexandridis, T.A. Hatton, Colloids Surfaces A Physicochem. Eng. Asp. 96 (1995) 1-46. DOI: doi:10.1016/0927-7757(94)03028-X

[6] P.C. Griffiths, A.Y.F. Cheung, G.J. Finney, C. Farley, S.M. King, R.K. Heenan, B.L. Bales, Langmuir 18 (2002) 1065-1072. DOI: 10.1021/la011163j

[7] J. Nambam, J. Philip, J. Phys. Chem. B 116 (2012) 1499-1507. DOI: 10.1021/jp208902a

[8] Y. Li, R. Xu, D.M. Bloor, J.F. Holzwarth, E. Wyn-Jones, S. Couderc, Langmuir 16 (2000) 10515-10520. DOI: 10.1021/la000899y

[9] R. Ivanova, B. Lindman, P. Alexandridis, Adv. Colloid Interface Sci. 89-90 (2001) 351-382. DOI: 10.1016/S0001-8686(00)00049-X

[10] A. Kabanov, E. Batrakova, V. Alakhov, J. Control. Release 82 (2002) 189-212. DOI: 10.1016/S0168-3659(02)00009-3

[11] A. Jangher, P.C. Griffiths, A. Paul, S.M. King, R.K. Heenan, R. Schweins, Colloids Surfaces A Physicochem. Eng. Asp. 391 (2011) 88-94. DOI: 10.1016/j.colsurfa.2011.08.006

[12] P. Linse, M. Malmsten, Macromolecules (1992) 5434-5439. DOI: 10.1021/ma00046a048

[13] P. Griffiths, N. Hirst, A. Paul, S. King, Langmuir (2004) 6904-6913. DOI: 10.1021/la049348o

[14] K. Nakashima, P. Bahadur, Adv. Colloid Interface Sci. 123-126 (2006) 75-96. DOI: $10.1016 /$ j.cis.2006.05.016

[15] R. Ganguly, V. Aswal, Langmuir 20 (2006) 9843-9849. DOI: 10.1021/jp0607061

[16] E. Hecht, K. Mortensen, J. Phys. Chem. B 88 (1995) 4866-4874. DOI: 10.1021/j100013a068

[17] C. Sarmoria, S. Puvvada, D. Blankschtein, Langmuir 8 (1992) 2690-2697. DOI: 10.1021/la00047a019 
[18] S.B.. Puvvada, Surfactants Solut. 11 (1991) 95-111.

[19] P. Griffiths, A. Paul, R. Heenan, J. Phys. Chem. B 108 (2004) 3810-3816. DOI: 10.1021/jp0371478

[20] P. Desai, N. Jain, R. Sharma, P. Bahadur, Colloids Surfaces A Physicochem. Eng. Asp. 178 (2001) 57-69. DOI: 10.1016/S0927-7757(00)00493-3

[21] B. Sarkar, V. Ravi, P. Alexandridis, J. Colloid Interface Sci. 390 (2013) 137-46. DOI: $10.1016 /$ j.jcis.2012.09.028

[22] B. Hammouda, Eur. Polym. J. 46 (2010) 2275-2281. DOI: 10.1016/j.eurpolymj.2010.10.012

[23] J. Hansen, J.B. Hayter, Mol. Phys. 42 (1981) 109-118. DOI: $10.1080 / 00268978100100091$

[24] J. Hansen, J.B. Hayter, Mol. Phys. 46 (1982) 651-656. DOI: 10.1080/00268978200101471

[25] SasView, Http://www.sasview.org/version 2.1.

[26] J. Joshi, V. Aswal, P. Goyal, Pramana 71 (2008) 1039-1043. DOI: 10.1007/s12043008-0220-z

[27] P. Occhipinti, P.C. Griffiths, Adv. Drug Deliv. Rev. 60 (2008) 1570-82. DOI: 10.1016/j.addr.2008.08.006

[28] P. Stilbs, K. Paulsen, P. Griffiths, J. Phys. Chem. B 3654 (1996) 8180-8189. DOI: 10.1021/jp9535607

[29] P. Stilbs, J. Colloid Interface Sci. 89 (1982) 547-554. DOI: 10.1016/00219797(82)90206-5

[30] A. Cifuentes, J. Bernal, J. Diez-Masa, Anal. Chem. 69 (1997) 4271-4274. DOI: 10.1021/ac970696n

[31] S. Lin, Y. Lin, E. Chen, C. Hsu, C. Kwan, Langmuir (1999) 4370-4376. DOI: 10.1021/la001075j

[32] B.D.Flockhart, J. Colloid Interface Sci. 565 (1957) 557-565.

[33] J. Bhattacharjee, G. Verma, V.K. Aswal, V. Patravale, P. a. Hassan, RSC Adv. 3 (2013) 23080-23089. DOI: 10.1039/c3ra44983a 
[34] S. Alexander, T. Cosgrove, T.C. Castle, I. Grillo, S.W. Prescott, J. Phys. Chem. B 116 (2012) 11545-11551. DOI: 10.1021/jp303185m

[35] P. Alexandridis, L. Yang, Macromolecules 33 (2000) 5574-5587. DOI: 10.1021/ma000332o

[36] S. Kumar, V.K. Aswal, J. Phys. Condens. Matter 23 (2011) 035101. DOI: 10.1088/0953-8984/23/3/035101

[37] V. Patel, J. Dey, R. Ganguly, S. Kumar, S. Nath, V.K. Aswal, P. Bahadur, Soft Matter 9 (2013) 7583. DOI: 10.1039/c3sm50600b

[38] T. Slawecki, C. Glinka, B. Hammouda, Phys. Rev. E 58 (1998) 4084-4087. DOI: DOI: http://dx.doi.org/10.1103/PhysRevE.58.R4084

[39] J. Pedersen, C. Svaneborg, Curr. Opin. Colloid Interface Sci. 7 (2002) 158-166. DOI: doi:10.1016/S1359-0294(02)00044-4

[40] S. Soni, G. Brotons, J. Phys. Chem. B 110 (2006) 15157-15165. DOI: $10.1021 /$ jp062159p

[41] S. Manet, A. Lecchi, J. Phys. Chem. B 115 (2011) 11318-11329. DOI: $10.1021 / j p 200212 \mathrm{~g}$

[42] J. Jansson, K. Schillén, J. Phys. Chem. B 109 (2005) 7073-7083. DOI: 10.1021/jp0468354

[43] E. Hecht, H. Hoffmann, Langmuir 14 (1994) 86-91. DOI: 10.1021/la00013a013

[44] S. Couderc, Y. Li, D.M. Bloor, J.F. Holzwarth, Langmuir 77 (2001) 4818-4824. DOI: $10.1021 / 1 \mathrm{a} 0104267$

[45] D. Lơf, M. Tomšrc, J. Phys. Chem. B 113 (2009) 5478-5486. DOI: doi/abs/10.1021/jp808442d

[46] M. Schwuger, J. Colloid Interface Sci. 43 (1973). DOI: 10.1016/00219797(73)90395-0

[47] J. Mata, T. Joshi, D. Varade, G. Ghosh, P. Bahadur, Colloids Surfaces A Physicochem. Eng. Asp. 247 (2004) 1-7. DOI: 10.1016/j.colsurfa.2004.07.011 